\title{
Affinity maturation of anti-TNF-alpha ScFv with somatic hypermutation in non-B cells
}

\author{
Shaopeng Chen ${ }^{1,2^{*}}$, Junkang Qiu ${ }^{1^{*}}$, Chuan Chen ${ }^{1^{*}}$, Chunchun Liu ${ }^{1,3}$, Yuheng Liu ${ }^{1}$, Lili An ${ }^{1}$, Junying Jia ${ }^{3}$, \\ Jie Tang ${ }^{1}$, Lijun $\mathrm{Wu}^{2}$, Haiying Hang ${ }^{1 凶}$ \\ ${ }^{1}$ Key Laboratory for Protein and Peptide Pharmaceuticals, National Laboratory of Biomacromolecules, Institute of Biophysics, \\ Chinese Academy of Sciences, Beijing 100101, China \\ ${ }^{2}$ Key Laboratory of Ion Beam Bioengineering, Institute of Technical Biology and Agriculture Engineering, Chinese Academy of \\ Sciences, Hefei 230031, China \\ ${ }^{3}$ Core Facility Center, Institute of Biophysics, Chinese Academy of Sciences, Beijing 100101, China \\ $\square$ Correspondence: ljw@ipp.ac.cn (L. Wu), hh91@ibp.ac.cn (H. Hang) \\ Received December 20, 2011 Accepted February 4, 2012
}

\section{ABSTRACT}

Activation-induced cytidine deaminase (AID) is required for the generation of antibody diversity through initiating both somatic hypermutation (SHM) and class switch recombination. A few research groups have successfully used the feature of AID for generating mutant libraries in directed evolution of target proteins in $B$ cells in vitro. B cells, cultured in suspension, are not convenient for transfection and cloning. In this study, we established an AID-based mutant accumulation and sorting system in adherent human cells. Mouse AID gene was first transfected into the human non-small cell lung carcinoma H1299 cells, and a stable cell clone (H1299-AID) was selected. Afterwards, anti-hTNF- $\alpha$ scFv (ATscFv) was transfected into H1299-AID cells and ATscFv was displayed on the surface of H1299-AID cells. By 4-round amplification/flow cytometric sorting for cells with the highest affinities to hTNF-alpha, two ATscFv mutant gene clones were isolated. Compared with the wild type ATscFv, the two mutants were much more efficient in neutralizing cytotoxicity of hTNF-alpha. The results indicate that directed evolution by somatic hypermutation can be carried out in adherent non-B cells, which makes directed evolution in mammalian cells easier and more efficient.

KEYWORDS antibody, activation-induced cytidine deaminase (AID), somatic hypermutation, affinity maturation, TNF-alpha

\section{INTRODUCTION}

Somatic hypermutation (SHM) of the $\mathrm{V}$ region of antibody genes confers much of antibody diversity and affinity maturation (Di Noia and Neuberger, 2007). Activation-induced cytidine deaminase (AID) is essential for the DNA cleavage that initiates SHM of the immunoglobulin (Ig) genes in B cells (Shivarov et al., 2008; Qiu and Hang, 2010). Mice and humans deficient in AID are incapable of somatic hypermutation and class switch recombination (Muramatsu et al., 2000). AID deaminates cytidine and causes mutations in the target DNA to produce secondary antibody repertoire in vivo. Taking advantage of the feature of AID, some immortal $B$ cell lines expressing AID were used to generate antibody libraries (Seo et al., 2006; Todo et al., 2006). Neuberger and his colleagues screened anti-streptavidin IgM and anti-protein A IgM from Ramos cells (human B cells) and DT 40 cells (chicken B cells) with 19-rounds and 14-rounds sorting, respectively (Cumbers et al., 2002).

Up to now, though AID expression in many cell types can induce SHM (Yoshikawa et al., 2002), in all reported studies directed protein evolution by SHM was only operated in B cells. However, gene manipulation is not easy in B cell lines. Some mammalian proteins expressing in different mammalian cell lines may have differential post-translational modification such as differential phosphorylation (Delgado et al., 1999) or glycosylation (Suriano et al., 2005). Therefore it is important to develop non-B cell line SHM evolution system to meet the demand of improving different target proteins' func-

\footnotetext{
*These authors contributed equally to the work.
} 
tions. According to our previous results and others' reports, human non-small cell lung carcinoma cell line H1299 cells were easy to be transfected, and could tolerate the high level expression of exogenous proteins and the stress of flow cytometry sorting (Jee et al., 2006; Windhorst et al., 2008). In this study, we intended to establish a non-B cell line SHM evolution system in $\mathrm{H} 1299$ cells by overexpression of AID. To do that, a mouse AID gene and an anti-hTNF- $\alpha$ scFv (ATscFv/wt) gene were expressed in $\mathrm{H} 1299$ cells. After 4 rounds of flow cytometric sorting, we obtained high-affinity mutants with more than 10-fold neutralization efficiency of the wild type antibody, and established a platform of evolving proteins on the surface of non-B cells with exogenous AID.

\section{RESULTS}

\section{Generation of a stable non-B cell line expressing AID for hypermutation}

To establish an autonomously diversifying library of mutants, the $\mathrm{pCl}-\mathrm{mAID}$ and pcDNA3.1/Hyg(+) were co-transfected into H1299 cells. The clones which highly expressed mouse AID were screened and identified with Western blotting by anti-AID antibody. As shown in Fig. 1A, the expression of AID in selected H1299-AID clones was several fold higher than that in Ramos cells (a hypermutation human B cell line). Since the AID used for constructing the cell clones was of mouse version and the anti-AID antibody might bind the mouse and Ramos human versions with very different efficiencies, we transfected Flag-tagged mouse and human AIDs to 293 cells and the lysates were subjected to Western blotting and probed with anti-Flag and anti-AID (L7E7) antibodies. The antibody (L7E7) binded the mouse AID 25\% more efficiently than the human AID. The cell line H1299-AID expressed AID 4.5 times more than Ramos cells based on the binding efficiency difference of the antibody to mouse AID and human AID and density quantification (data not shown). Therefore, mouse AID was highly overexpressed in H1299-AID cells compared to Ramos cells and H1299-AID cells were appropriate to use for mutating and screening
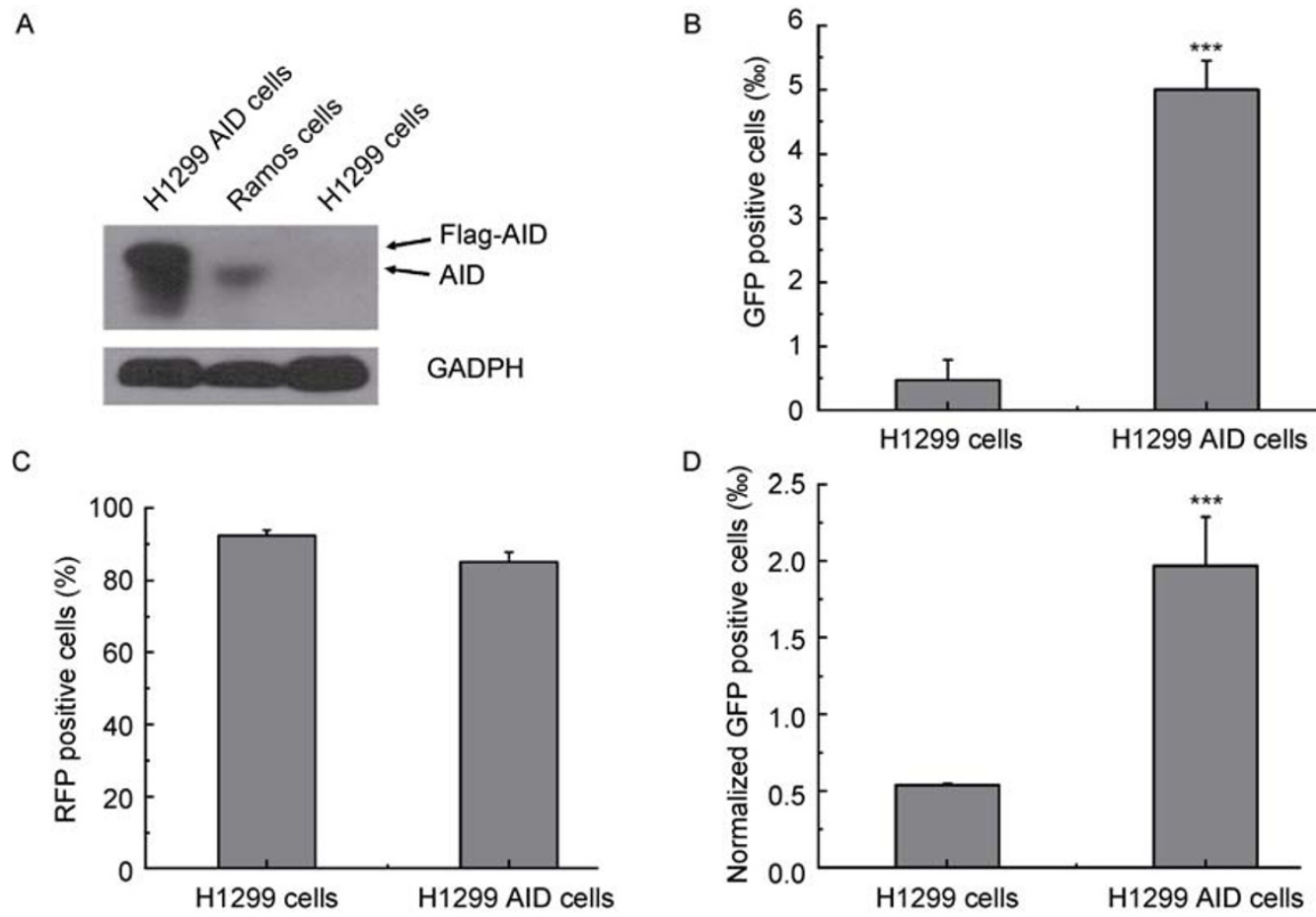

Figure 1. Establishment of H1299-AID cells for the in vitro evolution of target genes. (A) The expression of AID in a selected clone. The AID expression levels of the Ramos B cells, H1299-AID and H1299 cells were detected by Western blotting. The AID expression in $\mathrm{H} 1299$ cells is much higher than Ramos cells, and the parental H1299 cells have no AID expression. (B) Detection of hypermutation induced by AID in H1299-AID cells. The plasmid carrying a mutant GFP gene was transfected into H1299-AID and H1299 cells. After culturing for 2 days, the GFP fluorescence (reverse mutation) was determined by flow cytometry. The data are pooled from three independent experiments. (C) Detection of transfection efficiency of RFP gene. The plasmids carring RFP gene were transfected into H1299 and H1299-AID cells. Two days later, the RFP fluorescence was determined by flow cytometry. (D) Detection of hypermutation induced by AID in H1299-AID cells with GFP/RFP experiments. The plasmids carrying RFP and mutant GFP gene were co-transfected into $\mathrm{H} 1299$ and H1299-AID cells. After culturing for 2 days, the GFP fluorescence (reverse mutation) and RFP fluorescence were determined by flow cytometry. The results indicate that the TAG codon in H299-AID cells reverted significantly more frequently than that in $\mathrm{H} 1299$ cells after GFP signal was normalized with RFP signal. The data are pooled from three independent experiments. 


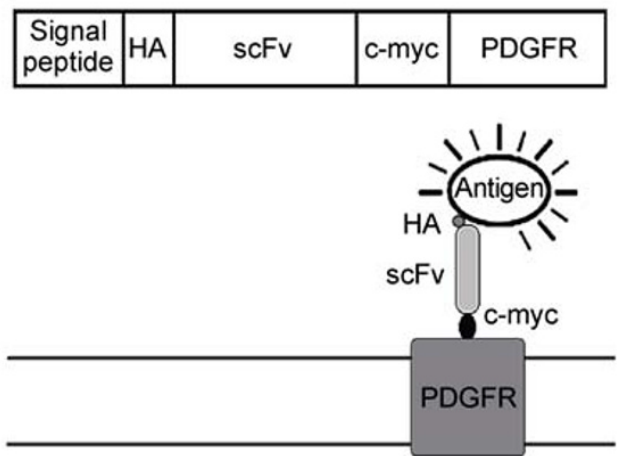

Mammalian cell plasma membrane
B
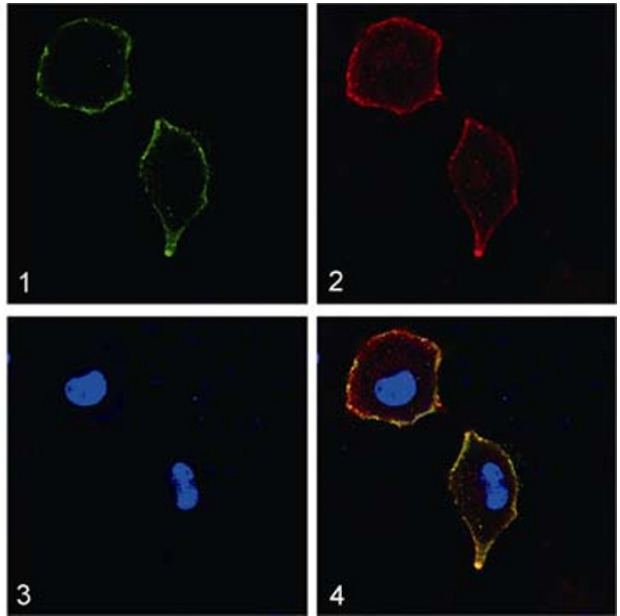

Figure 2. Displaying ATscFv/WT on the surface of H1299-AID cells. (A) Schematic diagram to illuminate mammalian cell surface display system and interaction with antigen (GFP-hTNF- $\alpha$ ). The upper panel shows the construct of displayed antibody gene with HA and cMyc tags. The lower panel shows that the recombinant antibody is displayed on the cell surface and the antigen (GFP-hTNF- $\alpha$ ) is bound to the top of the antibody. In this study the HA tag was used to monitor the level of display by anti-HA-PE. (B) Microscopic images of cells displaying ATscFvs. The cells were stained with GFP-hTNF- $\alpha$ (green, B1) and anti-HA-PE (red, B2) were used to label ATscFv and HA-tag, respectively. The cells were also stained with Hoechst 33258 (blue, B3) to locate the nuclei. Merged cell images clearly show that the recombinant antibodies were located on the cell surface.

high-affinity TNF- $\alpha$ mutant antibodies.

The effect of H1299-AID cells on somatic hypermutation was assessed by transfection of plasmids carrying a mutant GFP into cells. The mutant GFP contained a premature TAG stop codon, and was subcloned into EcoRl-Xbal digested pcDNA3.1(+) (Yoshikawa et al., 2002; Wang et al., 2004a). Figure 1B shows that the TAG codon reverted significantly more frequently in H1299-AID cells than that in H1299 cells $(5.0 \%$ vs $0.47 \%, P<0.001)$. To exclude the possibility that the mutation rate difference might be caused by different transfection efficiencies between $\mathrm{H} 1299$ and H1299-AID cells, we co-transfected two cell lines with mutant GFP and the wild type RFP genes simultaneously ( $\mathrm{He}$ et al., 2008). The transfection efficiencies of RFP to $\mathrm{H} 1299$ and H1299-AID were not much different $(92.4 \%$ and $85.1 \%$, respectively, shown in Fig. $1 \mathrm{C})$, and the reversion mutation rates normalized to RFP in $\mathrm{H} 1299$ and H1299-AID were $0.54 \%$ and $1.9 \%$ (the average of three repeated experiments, shown in Fig. 1D), respectively. Therefore, the TAG codon in H299-AID cells reverted significantly more than that in $\mathrm{H} 1299$ cells but lower than that when the only mutant GFP was transfected into the cells. It is known that AID-induced mutation requires high level of transcription of target gene (Sohail et al., 2003). The very intense transcription of RFP would compete with the transcription of mutant GFP, thus lowering the mutation efficiency of mutant GFP induced by AID. Also, the RFP expressions in both H1299 and H1299-AID did not differ much, excluding the possibility that the transfection efficiency difference caused mutation difference in the two cell lines. Therefore, an H1299-AID cell line was established and hypermutation can be induced in this cell line.

The core issue of the AID driven artificial evolution is whether AID has an added value in a background mutation $(0.47 \%$ in the current system). We sequenced GFP clones isolated from AID-expressing and control H1299 cells (cultured for 15 days), separately. Out of 46 GFP clones isolated from AID-expressing $\mathrm{H} 1299$ cells, 4 clones have point mutations and all these mutations are either $\mathrm{G} \rightarrow \mathrm{T}$ or $\mathrm{C} \rightarrow \mathrm{A}$, characteristic mutations induced by AID. The total point mutation sites are 9 . One clone has 5 point mutations. One of the mutations is an insertion. Taken together, the mutation frequency is $720 \times 10 / 46=3.0 \times 10^{-4} \mathrm{bp} / 15 \mathrm{~d}$. Only one point mutation was identified by sequencing 29 GFP clones isolated from control $\mathrm{H} 1200$ cells and the mutation frequency is $720 \times 1 / 29=4.79 \times 10^{-5} \mathrm{bp} / 15 \mathrm{~d}$.

It is noteworthy that we found $0.47 \%$ GFP-reversion mutation background in $\mathrm{H} 1299$ cells contained no AID and Yoshikawa et al. (2002) demonstrated that there was no GFP-reversion mutation in NIH3T3 murine fibroblast cells. The difference could be because we used different types of cells. NIH3T3 murine fibroblast cells are a relatively normal cell, while human non-small cell lung carcinoma H1299 cells are highly genomically unstable, thus causing autonomous mutations.

\section{Display of ATscFv/wt on the surface of AID-expressing cells}

In order to screen and sort for cells expressing ATscFv by a flow cytometer, the ATscFv protein needed to be displayed on the surface of cells. The plasmid pUHD10-3-dis/scFv was constructed by fusing ATscFv/wt in between signal peptide and PDGFR (Refer to MATERIALS AND METHODS; Fig. 2A). After co-transfecting pTet-off and pUHD10-3-dis/scFv 
vectors into H1299-AID cells, the ATscFv/wt was expressed on the surface of the H1299-AID cells in the absence of doxycycline. The plasmid pTet-off contains the neo-resistant gene for selection of positive cells with G418. As shown in Fig. 2B, GFP-hTNF- $\alpha$ (green) and anti-HA-PE antibody (red) were co-localized on the surface of the cells very well. This indicates that ATscFv/wt was efficiently displayed on the surface of cells.

\section{Isolation of high-affinity mutants from autonomously diversifying cell libraries}

For our purpose and the ease to carry out the in vitro evolution, we did not intentionally isolate a cell clone bearing a single ATscFv copy. Our flow cytometric sorting will pick one or more winner clones in a multi-step evolution process even though the winner clone is one of the multiple clones in a cell. In the end of the evolution process, we will isolate, sequence the potential winner gene clones, compare the antigen-binding abilities of their corresponding proteins with that of the original antibody, and eventually confirm the winner clones (Fig. 4). To evolve the target antibody, the positive AID-tet-scFv cells were enriched by flow cytometric sorting with a single color sorting model (Fig. 3A). High level expression of a target gene is required for a high mutation frequency induced by AID (Sohail et al., 2003). We tested a few cell lines including Hela, H1299 and 293T cells for expressing high levels of the GFP and antibody genes; H1299 was the most tolerable. Indeed, even it was the most tolerable, multiple enrichments were still necessary. Similar situation was encountered by Wang et al. (2004b). In addition, post-sorting survival is a tough issue (Wang and Tsien, 2006). It is possible that the in vitro evolution is not only to evolve the antibody gene, but also evolve the host cells against the challenges of high level expression of the antibody and flow cytometry sorting.

After the positive cells expressing ATscFv/WT were enriched to about $60 \%$, the sorting for directed evolution began. As shown in Fig. 3B (left and middle panels), less than $0.5 \%$ of the population in the sorting window with the strongest fluorescence was collected. After each round sorting, the cells were cultured for 10 days in the absence of doxycycline in which condition ATscFv was highly expressed. After each sorting-amplification round, the cells were labeled with GFP-hTNF- $\alpha$ and anti-HA-tag-PE, and examined by two-color histogram to monitor if there were cells with higher affinities emerged. Two-color histogram can help judge the affinity difference at similar levels of antibody expression. After the second-round sorting, the cells displaying antibodies with relative higher affinities to TNF- $\alpha$ appeared, in which the expression levels of ATscFvs were unaltered (Fig. 3C; $5.1 \%$ vs $1.0 \%$ ). After third-round sorting, the cells displaying antibodies with relative higher affinities to TNF- $\alpha$ increased to $13.2 \%$ while $0.7 \%$ in parallel control group of $\mathrm{H} 1299$ cells
(Fig. 3D). At fourth-round sorting, the cells were labeled with GFP-hTNF- $\alpha$ and anti-HA-tag-PE, and sorted in two-color histogram (Fig. 3B; right panel). These cells in the sorting window (S4) which expressed ATscFvs with higher affinities than that in other region were collected.

To confirm cells in the sorting window bearing ATscFv with higher affinities, the genes of ATscFv extracted from S4 cells were cloned and transfected into HEK 293 cells. As shown in Fig. 4A, cells expressing S4 scFv antibodies had higher affinities to the antigen than those cells expressing ATscFv/WT. By sequencing S4 scFv genes, two mutants were found (59.2\% of wild type, $32.7 \%$ of mutant 1 , and the rest of mutant 2). Figure 4B shows that mutant 2 (Mut2) had stronger binding abilities to the antigen than the wild type. The antigen binding ability of mutant 1 (Mut1) was slightly lower than that of mutant 2 (data not shown). To confirm these two mutants were specific to hTNF- $\alpha$, not GFP, the purified GFP was used to label 293T cells expressing ATscFv mutants. Determined by flow cytometry, GFP was not found to bind onto the surface of the cells (Data not shown). The results indicate that the high-affinity and specific ATscFvs were acquired by four rounds of cell amplification and flow cytometry sorting.

\section{Measurement of the affinity of surface-displayed ATscFvs}

The affinity of ATscFvs displayed on the cell surface was analyzed by flow cytometry (Refer to MATERIALS AND METHODS). The vectors containing ATscFvs/WT, ATscFv/Mut1, and ATscFv/Mut2 were transiently transfected into HEK 293T cells, respectively. Two days later, cells were harvested and labeled with designated concentrations of GFP-hTNF- $\alpha$. Figure 5 shows the results of two independent titrations of cell surface-displayed ATscFv. The mean fluorescence intensity of GFP-hTNF- $\alpha$ was normalized to the mean value of saturated fluorescence intensity of GFP-TNF- $\alpha$, and dissociation constant $(\mathrm{Kd})$ was calculated by fitting non-linear least squares. The Kd values of Mut1 and Mut2 were $3.86 \mathrm{nmol} / \mathrm{L}$ and $3.16 \mathrm{nmol} / \mathrm{L}$, respectively. The affinity of Mut2 was about 5.8-fold higher than that of WT (18.2 $\mathrm{nmol} / \mathrm{L})$. The affinity of ATscFv was successfully improved by the SHM system in H1299 cells.

\section{Improved potency of the mutants to neutralize GFP-hTNF- $\alpha$-induced cytotoxicity in L929 cells}

hTNF- $\alpha$ is highly toxic to $L 929$ cells. In this study, $0.1 \mathrm{nmol} / \mathrm{L}$ GFP-hTNF- $\alpha$ killed all of L929 cells after $16 \mathrm{~h}$ incubation. ATscFv could neutralize GFP-hTNF- $\alpha$-induced cytotoxicity via the blockade of the binding of GFP-hTNF- $\alpha$ to its receptors. Figure $6 \mathrm{~A}$ shows that the two mutants of ATscFv were much more efficient to attenuate the cytotoxicity of GFP-hTNF- $\alpha$ than the wild type antibody. The data in Fig. 6B demonstrate that $\mathrm{IC}_{40}$ of $\mathrm{ATscFv} /$ Mut1 and ATscFv/Mut2 were 16 -fold and 20 -fold more than ATscFv/WT, respectively. 

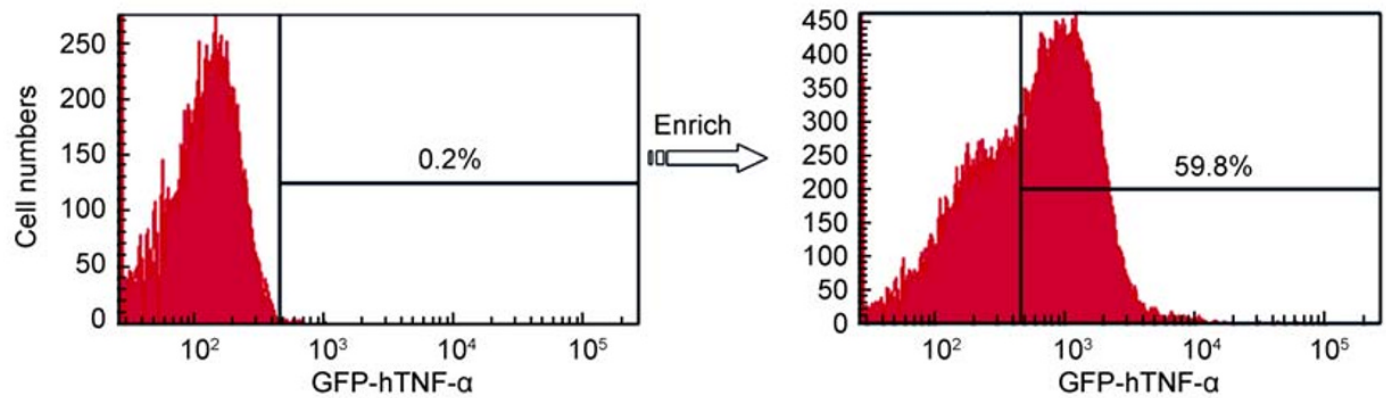

B
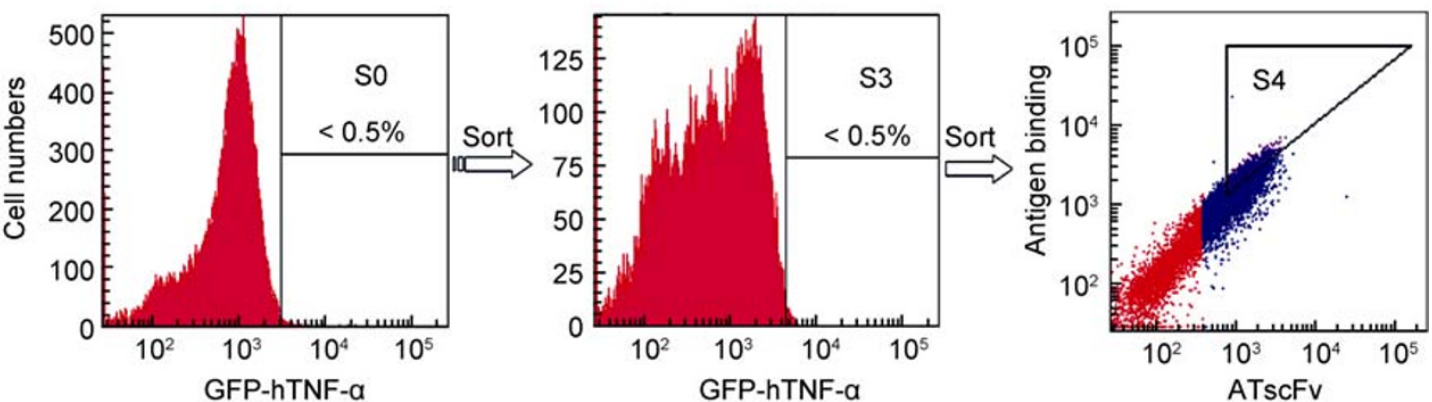

$\mathrm{C}$
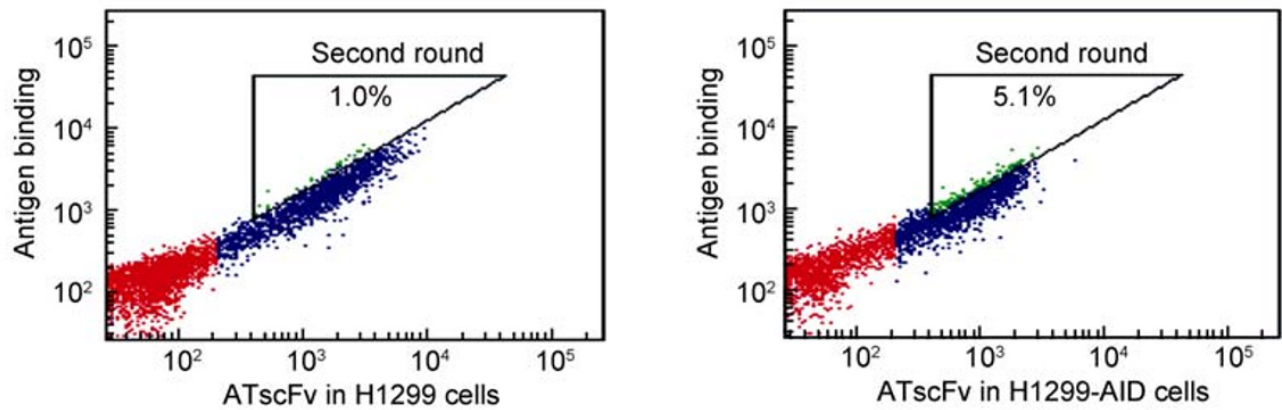

D
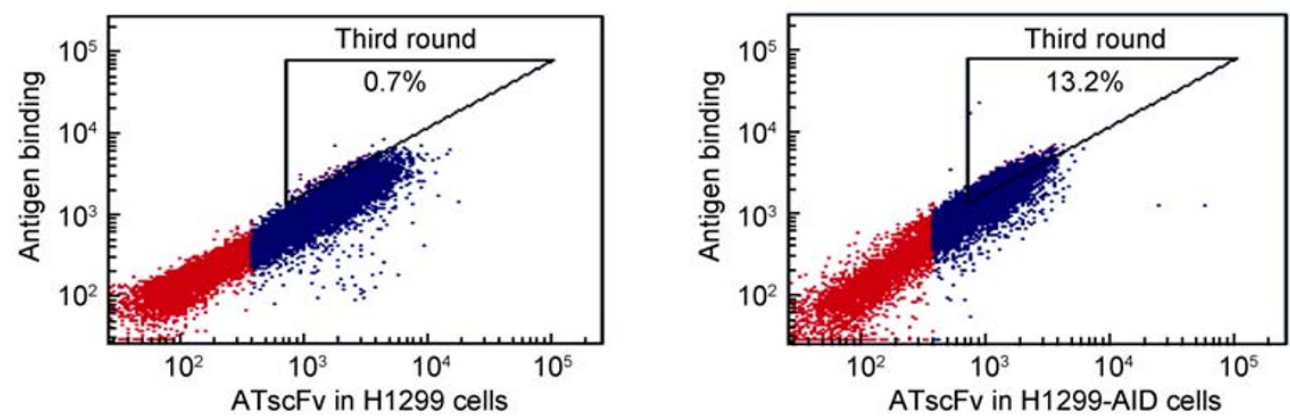

Figure 3. Directed evolution of ATscFv. (A) Enrichment of H1299 cells displaying ATscFv. The expression of ATscFv is tet-off controlled. Doxycycline was removed and the cells were cultured in doxycycline-free medium for two days, then flow-sorted for the cells displaying high levels of ATscFv. The ATscFv on the cell surface was labeled with GFP-hTNF- $\alpha(1 \mu \mathrm{g} / \mathrm{mL}$ in Optimal-MEM medium containing 5\% BSA). The enrichment was performed 9 times before the directed evolution. (B) Directed evolution of ATscFv. Doxycycline was removed and cells displaying high levels of ATscFv were cultured in doxycycline-free medium for 10 days, then subject to flow-sorting for cells bearing high affinity ATscFv. A total of 4 rounds of cell proliferation/flow sorting were performed. (C and D) The affinity maturation. (C) Left panel: Cells with the higher affinity in triangle region in H1299-tet-scFv cells after second-round sorting. Right panel: Cells with the higher affinity in triangle region in AID-tet-scFv cells after second-round sorting. (D) Left panel: Sorted cells with the higher affinity in triangle region in H1299-tet-scFv cells after third-round sorting. Right panel: Sorted cells with the higher affinity in triangle region in AID-tet-scFv cells after third-round sorting. 
A

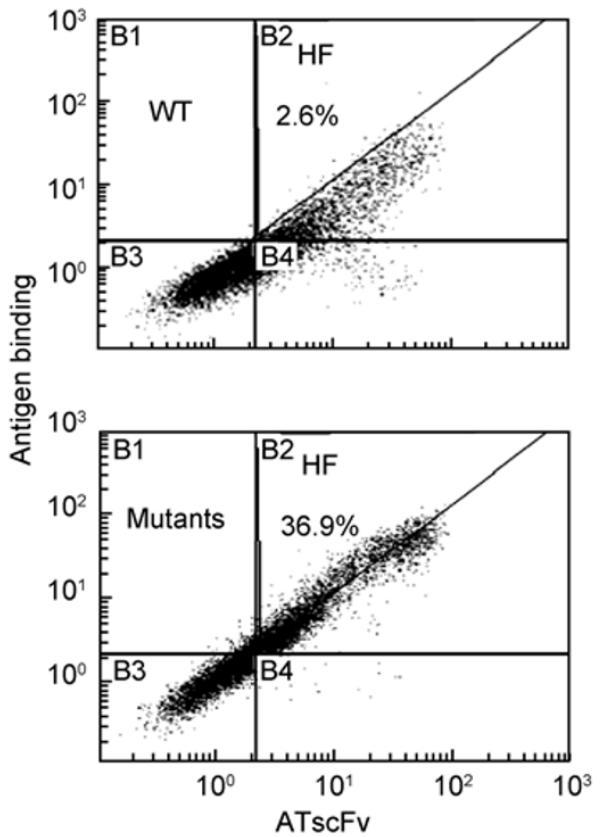

B

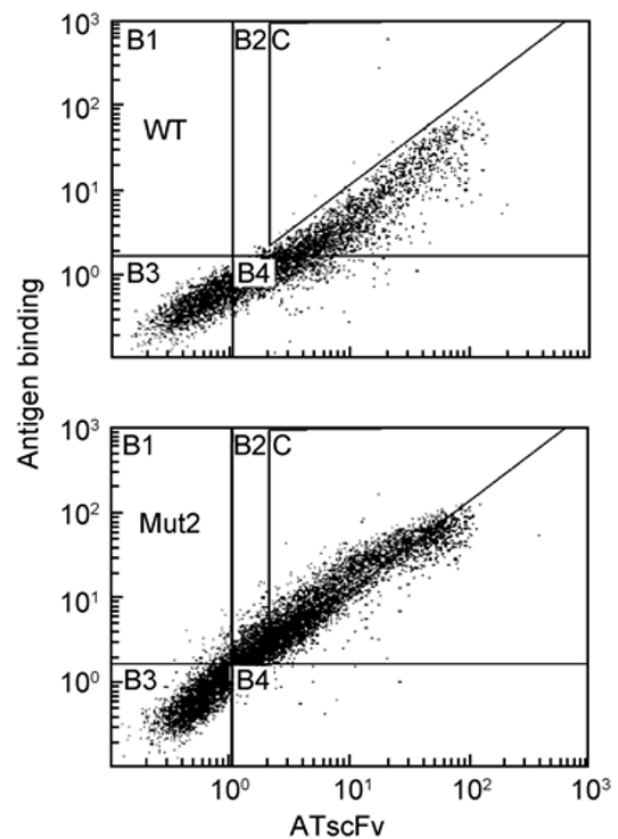

Figure 4. Determination of the mixed high-affinity evolved mutants. (A) Affinity of ATscFv genes cloned from fourth-round sorted cells. The ATscFv genes were cloned from the cells derived from the four rounds of directed evolution. The mixed clones were transfected into 293 cells, and the mixed ATscFv proteins displayed on the cell surface were monitored by flow cytometry. The wild type ATscFv was used as a negative control. (B) Affinity of ATscFv Mut2. Sequecing ATscFv gene clones revealed two mutants. They were measured as in (A) for their affinities to the antigen.

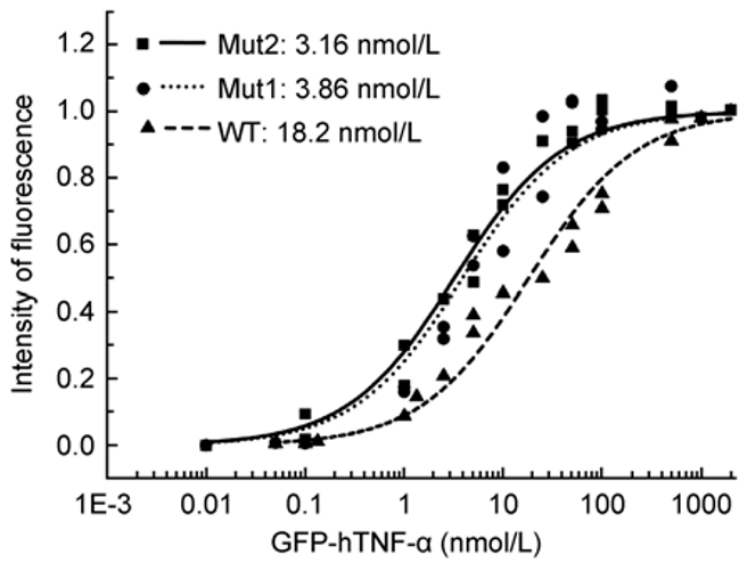

Figure 5. Affinities of isolated ATscFvs mutants. The binding constant Kd for scFvs specific for GFP-TNF- $\alpha$ on the cell surface was measured. The mean fluorescence intensity was plotted against designated concentrations of GFP-TNF- $\alpha$, and the data were fit to a monovalent binding isotherm with a non-linear least-squares regression.

These results indicate that the potency of neutralization of ATscFv/Mut was greatly improved compared with that of ATscFv/WT.

\section{DISCUSSION}

Since AID was discovered by Muramatsu et al. in 1999 (Mu- ramatsu et al., 1999), it attracted considerable attention of researchers for its essential role in affinity maturation of antibody. The evidence that expressing AID in non-B cells can also induce SHM indicates that AID itself is sufficient for the generation of SHM (Yoshikawa et al., 2002). Based on this and other similar observations, the studies on evolving proteins with SHM were carried out, and AID was regarded as a promising new tool to directly generate mutant libraries in cells including mammalian cells.

In the present study, we generated an antibody repertoire in non-B cells by overexpressing mouse AID, and screened for high-affinity mutants of ATscFv. By four rounds of cell proliferation and flow cytometric sorting, two mutants with higher affinities were selected by our designed sorting strategy. The affinities of the mutants were improved more than 5 -fold, and the potency of neutralization was improved 15-20 fold. The studies on evolving proteins with B cells (Ramos, DT40) have been reported by several research groups (Cumbers et al., 2002; Wang et al., 2004b; Seo et al., 2005; Arakawa et al., 2008). However, there has been no public report on a directed evolution of proteins by overexpressing AID in non-B cells. Our current study is the first effort to publish an in vitro evolution system for proteins of interest by overexpressing AID in non-B cells.

Researchers have devoted a lot of time and energy to improve AID-dependent evolution system in B cells. B cells are a good expression system of evolution antibody. Ohta's 
A

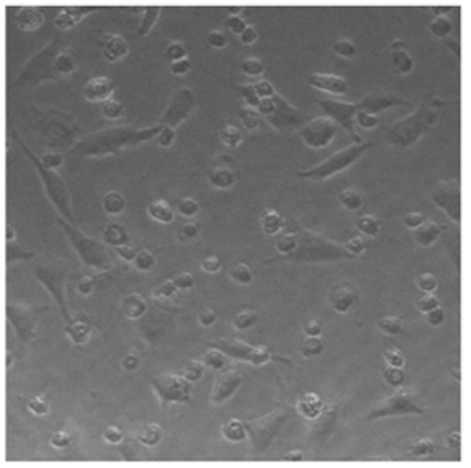

WT

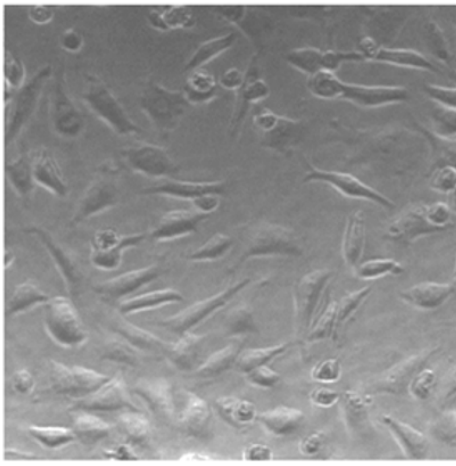

Mut1

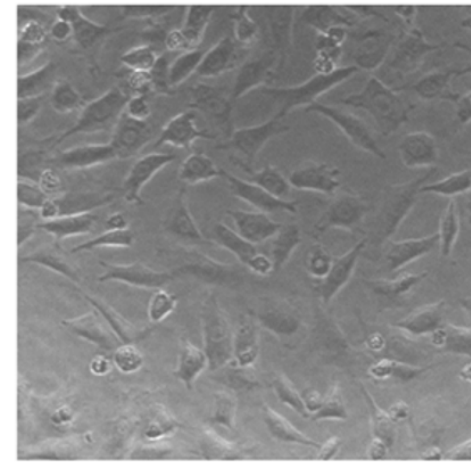

Mut2

B

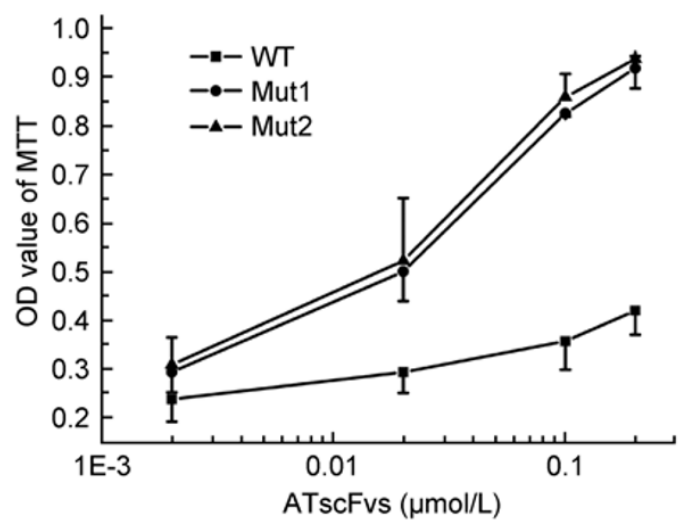

Figure 6. The competitive inhibitory effect of ATscFvs on GFP-hTNF- $\alpha$ in L929 cells. (A) Sample images of L929 cells which were treated with $0.1 \mathrm{nmol} / \mathrm{L}$ GFP-hTNF- $\alpha$ and $0.2 \mu \mathrm{mol} / \mathrm{L}$ ATscFvs. Cells were pretreated with ATscFv/WT, ATscFv/Mut1 and ATscFv/Mut2, then challenged with GFP-hTNF- $\alpha$. The cells pretreated with either ATscFv/Mut1 or ATscFv/Mut2 survived obviously better than those pretreated with the wild type ATscFv. (B) Determination of bioactivity of ATscFvs against GFP-hTNF- $\alpha$. The cell survival levels were assayed by MTT. The neutralization efficiency of ATscFv/WT, ATscFv/Mut1, and ATscFv/Mut2 were $30.4 \%$, $86.5 \%$, and $91.9 \%$, respectively. The data are pooled from three independent experiments.

group created an autonomously diversifying library (ADLib) system in DT40 cells, from which antigen-specific monoclonal antibodies can be rapidly generated (Seo et al., 2005). However, expression and/or modification of certain types of protein only happen in specific tissues and cells. In vitro evolution of such types of proteins in B cells are not always suitable for those proteins which need specific modification to maintain their functions in specifically defferentiated cells (Delgado et al., 1999; Suriano et al., 2005). Furthermore, evolving proteins with exogenous AID in non-B cells is more convenient for genetic manipulation to improve the mutation efficiency of AID or to regulate the expression of AID compared to the suspension-cultured $B$ cells. It was documented that some mutants of AID with increased catalytic activity gave more efficacious antibody diversification (Wang et al., 2009), and removal of nuclear export signal in its $C$ termini to locate AID in the nucleus also could increase AID-induced mutation efficiency (Ito et al., 2004). Substituting for the wild type AID with its mutants could increase the efficiency of AID to form diversity libraries efficiently. Furthermore, regulation of the expression of AID with tet-inducible promoter would decrease the risk of AID-induced genomic instability and increase the survival of cells screened by flow cytometric sorting. Inhibiting the expression of AID during certain periods would avoid unnecessary mutations and enhance cell survival. Therefore, the SHM evolution system in non-B cells described here is potentially advantageous over that of $B$ cells in directed evolution of protein in many aspects.

\section{MATERIALS AND METHODS}

\section{Construction of vectors}

To display antibodies on the surface of the cell, firstly, the primers of $\mathrm{P} 1 / \mathrm{P} 2$ (shown in Table 1) were used in PCR to clone the sequence including the $\lg \kappa$ chain leader, a hemagglutinin (HA) epitope tag, multiple cloning sites, a myc epitope tag and a transmembrane domain of human platelet-derived growth factor receptor (PDGFR) from the pDisplay (Invitrogen) into EcoRI-Xbal digested pUHD10-3 plasmid downstream of the tet response promotor (Gossen and Bujard, 1992) to yield pUHD10-3-dis. Then, the gene of ATscFv/wt (an anti-TNF- $\alpha$ antibody gene made by our group) was subcloned into Sfil-Sall digested pUHD10-3-dis to yield pUHD10-3-dis/scFv with 
primers of P3/P4 (Table 1). Anti-hTNF- $\alpha$ scFv (ATscFv) could be expressed on the surface of human cells via fusion to the PDGFR transmembrane domain at the C-terminal in the pUHD10-3-dis vector (Gronwald et al., 1988). For purification of scFv antibodies, secreting vectors (pUHD10-3-dis/scFv-sec) with his-tag were also constructed by replacing the PDGFR transmembrane domain.

Table 1 Primers used to amplify cDNA for constructing plasmids

\begin{tabular}{ll}
\hline Pimers & $\begin{array}{l}\text { Restriction } \\
\text { sites }\end{array}$ \\
\hline P1: 5' ACGCG/AATTCGCCACCATGGAGACAGACACACTCCTGCT 3' EcoRI \\
P2: 5' AACTAGT/CTAGACTAACGTGGCTTCTTCTGCCAAAGCAT 3' Xbal \\
P3: 5' CGCATTGGCCCAGC/CGGCCATGGATATCGGAAT 3' & Sfil \\
P4: 5' ATTCGCG/TCGACCTCGAGCCGTTTTA 3' & Sall \\
P5: 5' ACGCACGGATCCGTCAGATCATCTTCTCG 3' & BamHI \\
P6: 5' ATCTAGCTCGAGTCACAGGGCAATGATCCCAAAGTAG 3' & Xhol \\
P7: 5' ATTGCGGCTAGCATGGTGAGCAAGGGCGAG 3' & Nhel \\
P8: 5' ATTCGCGGATCCCTTGTACAGCTCGTCCA 3' & BamHI \\
\hline
\end{tabular}

To purify hTNF- $\alpha$ and GFP-hTNF- $\alpha$, the PCR product of $h T N F-\alpha$ gene was subcloned into $\mathrm{BamHI}$ and $\mathrm{Xhol}$ sites of $\mathrm{pET} 28 \mathrm{a}(+)$ vector (Novagen) to make the plasmid pET28a-hTNF- $\alpha$ with primers of P5/P6. Then, the GFP gene was fused into Nhel and BamHI sites of upstream of $h T N F-\alpha$ to finally construct the plasmid pET28a-GFPhTNF with primers of P7/P8.

\section{Cell culture}

Human non-small cell lung carcinoma H1299 cells and human embryonic kidney HEK293T cells were cultured in DMEM growth medium. Murine aneuploid fibrosarcoma L929 cells were maintained in RPMI-1640 growth medium. All of the growth media were prepared by basal media supplemented with $10 \%$ fetal bovine serum (FBS; Minhai Company), $100 \mathrm{U} / \mathrm{mL}$ penicillin and $100 \mathrm{~g} / \mathrm{mL}$ streptomycin (Gibco). All the cells were incubated at $37^{\circ} \mathrm{C}$ with $5 \% \mathrm{CO}_{2}$.

\section{Transfection and establishment of stable cell lines}

For generation of cell lines stably expressing AID, pCl-mAID (containing a flag epitope tag) (Wu et al., 2005) and pcDNA3.1/Hyg (+) were co-transfected into $\mathrm{H} 1299$ cells using the Lipofectamine 2000 kit (Invitrogen). Stable H1299-AID clones were selected in growth media supplemented with $150 \mu \mathrm{g} / \mathrm{mL}$ Hygromycin B (Invitrogen) for 10 days and identified by Western blotting with AID antibody (Cell Signaling Technology, Cat\#4975). This anti-AID antibody had enough crossreactivity to mouse AID. Then $50 \mu \mathrm{g} / \mathrm{mL}$ Hygromycin B was added into normal DMEM growth medium to maintain H1299-AID cells.

To display ATscFv/wt on the surface of H1299-AID cells, pUHD10-3-dis/scFv and pTet-off were co-transfected into H1299-AID cells. The display of ATscFv/wt can be controlled in a tet-off response manner. After removing the doxycycline to induce the expression of ATscFv/wt, the cells displaying ATscFv/wt were labeled with GFP-hTNF- $\alpha$ and sorted with enrichment model by flow cytometry. After sorting, the cells were proliferated in the growth media containing $100 \mu \mathrm{g} / \mathrm{mL}$ G418, $50 \mu \mathrm{g} / \mathrm{mL}$ Hygromycin B, and complemented with $1.5 \mu \mathrm{g} / \mathrm{mL}$ doxycycline to shut down the expression of ATscFv/wt.

\section{Preparation and purification of hTNF- $\alpha$, GFP-hTNF- $\alpha$ and scFv}

For the expression of hTNF- $\alpha$ or GFP-hTNF- $\alpha$, Rosetta cells (Invitrogen) harboring expression plasmid were cultured in LB broth containing $50 \mathrm{~g} / \mathrm{mL}$ kanamycin for His-hTNF- $\alpha$ or His-GFP-hTNF- $\alpha$ at $37^{\circ} \mathrm{C}$. Protein expression was induced at $0.5 \mathrm{OD}_{600}$ by the addition of isopropytthiogalactoside (IPTG) to a final concentration of $0.25 \mathrm{mmol} / \mathrm{L}$ and cells were grown at $16^{\circ} \mathrm{C}$ for $4 \mathrm{~h}$ and then harvested by centrifugation. The cell paste, from a $250-\mathrm{mL}$ culture, was resuspended in $10 \mathrm{~mL}$ PBS containing $1 \mathrm{mmol} / \mathrm{L}$ PMSF at $4^{\circ} \mathrm{C}$. After sonication and centrifugation at $16000 \mathrm{~g}$ for $30 \mathrm{~min}$, the supernatant was saved and added to a $50 \%$ slurry of Ni Sepharose High Performance column (Amersham Biosciences). To collect hTNF- $\alpha$ or GFP-hTNF- $\alpha$, the Ni Sepharose High Performance column was rinsed with $10,25,50,100$ and $250 \mathrm{mmol} / \mathrm{L}$ imidazole in binding buffer (500 mmol/L NaCl, 20 mmol/L Tris-Cl, 15\% glycerol, pH 7.5) in a stepwise manner. After purification, the concentration of these recombinant proteins was determined with Bradford protein assay kit (Bio-Rad) according to standard procedure. The purity of GFP-hTNF- $\alpha$ was more than $92 \%$ determined by SDS-PAGE.

For purification of scFv antibodies, $5 \mu \mathrm{g}$ pUHD10-3-dis/scFv-sec was transiently transfected into $5 \times 10^{6}$ HEK 293 T cells cultured in one $100-\mathrm{mm}$ Petri dish. Next day, the cells and media were transferred into a 145-mm Petri dish, and maintained for 4 days. The media were collected and scFv antibodies were purified as above. Afterwards, the concentration of $\mathrm{ScFv}$ antibodies was determined with Bradford protein assay kit as above. The purity of ScFv antibodies was about $82 \%$ determined by SDS-PAGE.

\section{Fluorescence microscopy}

Cells were inoculated on sterile cover slips. After cultured for $48 \mathrm{~h}$, cells were washed with PBS and fixed with $4 \%$ formaldehyde in PBS for $20 \mathrm{~min}$ at $25^{\circ} \mathrm{C}$. The fixed cells were permeabilized in TNBS solution (PBS supplemented with $0.1 \%$ Triton X-100 and $1 \%$ FBS), followed by exposure to anti-HA-PE antibody (ab72564, Abcam Ltd., 1:300 in PBS) and GFP-hTNF- $\alpha(1 \mu \mathrm{g} / \mathrm{mL}$ in PBS) for $25 \mathrm{~min}$. After washing with TNBS for 3-5 min, the cells were counterstained with Hoechst 33342 (Molecular Probes, Eugene, Oregon, USA) for $10 \mathrm{~min}$ at $25^{\circ} \mathrm{C}$. Slides were analyzed under an LSCMFV500 confocal microscope (Olympus).

\section{Assay and sorting with flow cytometry}

The expression of ATscFv is tet-off controlled. H1299-AID ATscFv cells were induced to display ATscFv by removing the doxycycline. The induced cells were collected by centrifugation at $200 \mathrm{~g}$ for $5 \mathrm{~min}$ and washed with PBS. Then the cells were incubated with anti-HA-PE antibody (Abcam, 1:300 in Optimal-MEM medium (Invitrogen) containing $5 \% \mathrm{BSA})$ and GFP-hTNF- $\alpha(1 \mu \mathrm{g} / \mathrm{mL}$ in Optimal-MEM medium (Invitrogen) containing $5 \% \mathrm{BSA}$ ) for 25 min at $4{ }^{\circ} \mathrm{C}$. After washed with cold PBS, the cells were resuspended in cold basal DMEM medium. The fluorescence associated with the living cells was detected by FACSCalibur (BD biosciences), or sorted by a FACSAriall cell sort (BD biosciences) with a sorting window as shown in Fig. 3B.

For in vitro evolution of ATscFv, firstly, the cells were flow-sorted 
with yield model. H1299-AID ATscFv cells were cultured in doxycycline-free medium for two days. When the positive cells expressing ATscFv were enriched to about $60 \%, \mathrm{H} 1299-A I D$ ATscFv cells were induced for 10 days in the absence of doxycycline, then the cells with high levels of ATscFv were sorted and collected with purified model. More than $10^{7}$ cells were examined and the sorting window was set to collect less than $0.5 \%$ of the population with top GFP fluorescence. Doxycycline was added to the sorted cells to stop the expression of ATscFv.

\section{Determination of affinity constants $\left(K_{d}\right)$ with cell surface display}

Antibody affinity measurements were performed with flow cytometry as described (Van Antwerp and Wittrup, 2000; Chao et al., 2006). Briefly, the exponentially growing cells transiently expressing ATscFv were dissociated from culture dishes with with trypsine solution, washed with PBS and divided into separate Eppendorf tubes. Then, cells in each tube were treated with designated concentration of GFP-hTNF- $\alpha$ as described above. After washed with cold PBS, the fluorescent intensity of cells was examined with Gallios flow cytometer (BackmanCoulter, Brea). The population of single cells was gated and their mean fluorescence intensity was recorded. The equilibrium dissociation constants were calculated by fitting the nonlinear curve with Origin software (version8.0987; OriginLab; Northampton.).

\section{Neutralization test}

The hTNF-sensitve murine L929 fibroblasts were used to evaluate the efficiency of ATscFv and its mutants for their abilities to neutralize the hTNF- $\alpha$ (Hogan and Vogel, 2001). A total of $100 \mu \mathrm{L}$ of L929 fibroblast suspension ( 300 cells $/ \mu \mathrm{L}$ ) was plated into 96 -well plate. Five hours later when the cells were adhered to the bottom of the plate, all media were aspirated from each well, and $100 \mu \mathrm{L}$ of test 1640 media containing $10 \%$ FBS, $2.5 \mu \mathrm{g} / \mathrm{mL}$ actinomycin D, $0.1 \mathrm{nmol} / \mathrm{L}$ GFP-hTNF- $\alpha$ and serially diluted anti-hTNF- $\alpha$ scFv was added to respective wells. Actinomycin D was used to sensitize L929 fibroblasts and increase hTNF- $\alpha$-induced apoptosis (Hogan and Vogel, 2001). After incubation for $16 \mathrm{~h}$, the supernatant was aspirated and $20 \mu \mathrm{L}$ methylthiazolyldiphenyl-tetrazolium bromide (MTT, Sigma) was added into each well. The cells were stained for $3 \mathrm{~h}$ in the incubator, the supernatant was aspirated and $150 \mu \mathrm{L}$ dimethyl sulfoxide (DMSO, Sigma) were added into each well. After agitating gently for $1 \mathrm{~min}$, OD value was detected by micaro-plated read at $570 \mathrm{~nm}$. Statistical analysis was performed on the means of the data pooled from at least three independent experiments.

\section{Statistical analysis}

The data were presented as means and standard derivations. The significance levels were assessed using Student's $t$-test. A $P$-value of 0.05 or less between groups was considered statistically significant.

\section{ACKNOWLEDGEMENTS}

This study was funded by grants from the Ministry of Science and Technology of People's Republic of China (Nos. 2011CBA00906 and
2011YQ03013404). We are grateful to BackmanCoulter China to allow us to use its Gallios flow cytometer to analyze cells in this study.

\section{ABBREVIATIONS}

AID, activation-induced cytidine deaminase; GFP, green fluorescent protein; Ig, immunoglobulin; SDS-PAGE, sodium dodecyl sulfate polyacrylamide gel electrophoresis; SHM, somatic hypermutation; RFP; red fluorescence protein; TNF- $\alpha$, tumor necrosis factor $\alpha$

\section{REFERENCES}

Arakawa, H., Kudo, H., Batrak, V., Caldwell, R.B., Rieger, M.A., Ellwart, J.W., and Buerstedde, J.M. (2008). Protein evolution by hypermutation and selection in the B cell line DT40. Nucleic Acids Res 36, e1.

Chao, G., Lau, W.L., Hackel, B.J., Sazinsky, S.L., Lippow, S.M., and Wittrup, K.D. (2006). Isolating and engineering human antibodies using yeast surface display. Nat Protoc 1, 755-768.

Cumbers, S.J., Williams, G.T., Davies, S.L., Grenfell, R.L., Takeda, S., Batista, F.D., Sale, J.E., and Neuberger, M.S. (2002). Generation and iterative affinity maturation of antibodies in vitro using hypermutating B-cell lines. Nat Biotechnol 20, 1129-1134.

Delgado, M.D., Chernukhin, I.V., Bigas, A., Klenova, E.M., and León, J. (1999). Differential expression and phosphorylation of CTCF, a c-myc transcriptional regulator, during differentiation of human myeloid cells. FEBS Lett 444, 5-10.

Di Noia, J.M., and Neuberger, M.S. (2007). Molecular mechanisms of antibody somatic hypermutation. Annu Rev Biochem 76, 1-22.

Gossen, M., and Bujard, H. (1992). Tight control of gene expression in mammalian cells by tetracycline-responsive promoters. Proc Natl Acad Sci U S A 89, 5547-5551.

Gronwald, R.G., Grant, F.J., Haldeman, B.A., Hart, C.E., O'Hara, P.J., Hagen, F.S., Ross, R., Bowen-Pope, D.F., and Murray, M.J. (1988). Cloning and expression of a cDNA coding for the human platelet-derived growth factor receptor: evidence for more than one receptor class. Proc Natl Acad Sci U S A 85, 3435-3439.

He, W., Zhao, Y., Zhang, C., An, L., Hu, Z., Liu, Y., Han, L., Bi, L., Xie, Z., Xue, P., et al. (2008). Rad9 plays an important role in DNA mismatch repair through physical interaction with MLH1. Nucleic Acids Res 36, 6406-6417.

Hogan, M.M., and Vogel, S.N. (2001). Measurement of tumor necrosis factor alpha and beta. Curr Protoc Immunol Chapter 6, Unit 6 10.

Ito, S., Nagaoka, H., Shinkura, R., Begum, N., Muramatsu, M., Nakata, M., and Honjo, T. (2004). Activation-induced cytidine deaminase shuttles between nucleus and cytoplasm like apolipoprotein B mRNA editing catalytic polypeptide 1. Proc Natl Acad Sci U S A 101, 1975-1980.

Jee, B.K., Park, K.M., Surendran, S., Lee, W.K., Han, C.W., Kim, Y.S., and Lim, Y. (2006). KAl1/CD82 suppresses tumor invasion by MMP9 inactivation via TIMP1 up-regulation in the H1299 human lung carcinoma cell line. Biochem Biophys Res Commun 342, 655-661.

Muramatsu, M., Kinoshita, K., Fagarasan, S., Yamada, S., Shinkai, Y., and Honjo, T. (2000). Class switch recombination and hypermutation require activation-induced cytidine deaminase (AID), a po- 
tential RNA editing enzyme. Cell 102, 553-563.

Muramatsu, M., Sankaranand, V.S., Anant, S., Sugai, M., Kinoshita, K., Davidson, N.O., and Honjo, T. (1999). Specific expression of activation-induced cytidine deaminase (AID), a novel member of the RNA-editing deaminase family in germinal center B cells. J Biol Chem 274, 18470-18476.

Qiu, J., and Hang, H. (2010). Mimicry of nature-Protein directed evolution based on DNA hypermutation. ACTA BIOP HYS ICA S INICA 26, 855-860.

Seo, H., Hashimoto, S., Tsuchiya, K., Lin, W., Shibata, T., and Ohta, K. (2006). An ex vivo method for rapid generation of monoclonal antibodies (ADLib system). Nat Protoc 1, 1502-1506.

Seo, H., Masuoka, M., Murofushi, H., Takeda, S., Shibata, T., and Ohta, K. (2005). Rapid generation of specific antibodies by enhanced homologous recombination. Nat Biotechnol 23, 731-735.

Shivarov, V., Shinkura, R., and Honjo, T. (2008). Dissociation of in vitro DNA deamination activity and physiological functions of AID mutants. Proc Natl Acad Sci U S A 105, 15866-15871.

Sohail, A., Klapacz, J., Samaranayake, M., Ullah, A., and Bhagwat, A.S. (2003). Human activation-induced cytidine deaminase causes transcription-dependent, strand-biased $\mathrm{C}$ to $\mathrm{U}$ deaminations. Nucleic Acids Res 31, 2990-2994.

Suriano, R., Ghosh, S.K., Ashok, B.T., Mittelman, A., Chen, Y., Banerjee, A., and Tiwari, R.K. (2005). Differences in glycosylation patterns of heat shock protein, gp96: implications for prostate cancer prevention. Cancer Res 65, 6466-6475.

Todo, K., Miyake, K., Magari, M., Kanayama, N., and Ohmori, H. (2006). Novel in vitro screening system for monoclonal antibodies using hypermutating chicken B cell library. J Biosci Bioeng 102, 478-481.

Van Antwerp, J.J., and Wittrup, K.D. (2000). Fine affinity discrimination by yeast surface display and flow cytometry. Biotechnol Prog $16,31-37$.

Wang, C.L., Harper, R.A., and Wabl, M. (2004a). Genome-wide somatic hypermutation. Proc Natl Acad Sci U S A 101, 7352-7356.

Wang, L., Jackson, W.C., Steinbach, P.A., and Tsien, R.Y. (2004b). Evolution of new nonantibody proteins via iterative somatic hypermutation. Proc Natl Acad Sci U S A 101, 16745-16749.

Wang, L., and Tsien, R.Y. (2006). Evolving proteins in mammalian cells using somatic hypermutation. Nat Protoc 1, 1346-1350.

Wang, M., Yang, Z., Rada, C., and Neuberger, M.S. (2009). AID upmutants isolated using a high-throughput screen highlight the immunity/cancer balance limiting DNA deaminase activity. Nat Struct Mol Biol 16, 769-776.

Windhorst, S., Blechner, C., Lin, H.Y., Elling, C., Nalaskowski, M., Kirchberger, T., Guse, A.H., and Mayr, G.W. (2008). Ins(1,4,5)P3 3-kinase-A overexpression induces cytoskeletal reorganization via a kinase-independent mechanism. Biochem J 414, 407-417.

Wu, X., Geraldes, P., Platt, J.L., and Cascalho, M. (2005). The double-edged sword of activation-induced cytidine deaminase. J Immunol 174, 934-941.

Yoshikawa, K., Okazaki, I.M., Eto, T., Kinoshita, K., Muramatsu, M., Nagaoka, H., and Honjo, T. (2002). AID enzyme-induced hypermutation in an actively transcribed gene in fibroblasts. Science 296, 2033-2036. 